\title{
Localization of Probabilistic Robot Formations in SLAM
}

\author{
M. T. Lázaro J. A. Castellanos \\ Instituto de Investigación en Ingeniería de Aragón, Universidad de Zaragoza (Spain) \\ Email: \{mtlazaro, jacaste\}@unizar.es
}

\begin{abstract}
This paper presents an EKF-based approach to the problem of robot formation pose tracking in SLAM when a previously built feature-based stochastic map of a navigation area is available. We show how a direct implementation of the EKF algorithm leads to inconsistency in the estimated localization. We justify the origin of the anomalous behaviour of the filter in the time-correlated nature of the measurement noise sequence. A novel solution based on the measurement differencing technique is proposed to drive the solution of the EKF towards consistency. Both simulation and real experiments with a 3-robot triangular-shaped formation are reported.
\end{abstract}

\section{INTRODUCTION}

Nowadays a big part of the robotics community is focused on the Simultaneous Localization and Mapping (SLAM) problem. We know how to build maps more and more accurately, efficiently and without requiring a big computational effort. A variety of representations for mapping indoor environments have been proposed throughout recent years including feature-based maps, grid-based maps and fuzzy maps.

The use of previously built maps to localize individual vehicles has been reported in [9] and [7] from a feature-based perspective, in [15] by using grid-based maps and [16] by combining grid-based and topological maps. Regarding to multi-robot cooperative localization, the works in [6] and [10] divided the team of robots into two groups: while one group moved, the other group remained stationary and acted as landmark. This strategy is useful in the absence of landmarks or in uncharted environments but the robots must maintain visual contact which constraints the robot displacements. Additionally in [14] inter-robot position measurements are used within an EKF framework to improve the estimates of the group and in [13] a distributed algorithm is proposed to localize a team of vehicles. However, and to the best of our knowledge, any reported work has considered the use of feature-based stochastic maps for solving the robot formation localization problem.

Additionally, and from a state estimation perspective, consistency issues are of paramount importance to assure convergence of the solution provided by the estimation algorithm. In [5] the consistency of multi-robot cooperative localization was investigated from an observability perspective. Also, the effect of using repeated measurements on the consistency of the algorithm has been reported in [8] by taking into

This work was partially supported by the European project IST-1-045062URUS-STP and the Spanish projects DPI2006-13758 and DPI2009-13710. account the correlations between consecutive relative-state measurements, and in [1] by keeping track of the origins of measurements and preventing them from being used more than once.

In the work reported in this paper, aligned with the general objectives of the European project URUS [18], we turn our attention towards using a previously built feature-based stochastic map to localize a team of autonomous vehicles while they navigate coordinately. We propose an algorithm to localize a team of robots within a stochastic map where each robot cooperates to obtain a more accurate and consistent position while navigating and avoiding obstacles.

Our contributions are two-fold. First, the work emphasizes the inherent difficulties of using stochastic maps to localize a robot formation due to the existence of time-correlated measurement sequences. Whenever these statistical dependencies are not properly considered we show that the algorithms lead to inconsistent estimation of the robot formation localization. Second, we originally formulate the problem of robot formation localization in SLAM within the EKF framework by using the measurement differencing technique [4], [2], [11] which allows the elimination of colored measurement sequences within the update step of the filtering algorithm.

This article is organized as follows: section II defines the probabilistic representation of the robot formation and presents the EKF formulation of the robot formation localization by assuming white noise measurement sequences. Simulation results illustrates the inconsistency of the approach. Then, section III proposes a modified EKF algorithm based on measurement differencing which correctly interprets the inherent time-correlated nature of the measurement sequence and it provides a consistent solution for the estimated robot formation localization. Section IV reports both simulation experiments within a rapid prototyping environment and real experiments with a 3-robot triangular-shaped formation of Pioneer 3-AT vehicles. Finally, section V summarizes the work and proposes future research directions.

\section{EKF-BASED LOCALIZATION OF THE ROBOT FORMATION}

Let a robot formation be composed of $r+1$ heterogeneous vehicles $\left\{R_{0}, R_{1}, \ldots, R_{r}\right\}$, where $R_{0}$ is the robot leader and $R_{j}, j=\{1, \ldots, r\}$ are the robot slaves. A certain geometric shape, e.g. equilateral triangle, regular pentagon, etc, is imposed to the team depending on the number of vehicles and the task commanded to the formation. 
From a probabilistic view-point, the location of the robot formation can be represented by a discrete-time state vector $\mathbf{x}_{\mathcal{R}}$ formed by the location of the robot leader $R_{0}$ with respect to (wrt) a base reference frame $B$ and the location of each robot slave $R_{j}$ wrt the robot leader $R_{0}$, and by its associated covariance matrix $\mathbf{P}_{\mathcal{R}}$ which stores the statistical dependencies between those estimated locations. Following the Gaussinity assumption, $\mathbf{x}_{\mathcal{R}} \sim \mathcal{N}\left(\hat{\mathbf{x}}_{\mathcal{R}}, \mathbf{P}_{\mathcal{R}}\right)$ with,

$$
\hat{\mathbf{x}}_{\mathcal{R}}=\left(\begin{array}{c}
\hat{\mathbf{x}}_{R_{0}}^{B} \\
\hat{\mathbf{x}}_{R_{1}}^{R_{0}} \\
\vdots \\
\hat{\mathbf{x}}_{R_{r}}^{R_{0}}
\end{array}\right) ; \mathbf{P}_{\mathcal{R}}=\left(\begin{array}{ccc}
\mathbf{P}_{R_{0}} & \cdots & \mathbf{P}_{R_{0} R_{r}} \\
& \ddots & \\
\mathbf{P}_{R_{r} R_{0}} & \cdots & \mathbf{P}_{R_{r}}
\end{array}\right)
$$

This leader-centric representation reduces the volume of uncertainty, i.e. the determinant of the covariance matrix $\mathbf{P}_{\mathcal{R}}$, in comparison with an absolute representation wrt the base frame $B$ of each robot location vector and, therefore, linearization errors due to large uncertainty values are minimized.

From a Bayesian view-point the pose of the robot formation is given by the recursively estimated conditional probability density function $p\left(\mathbf{x}_{\mathcal{R}_{k}} \mid \mathbf{y}_{E_{0: k}}, \mathbf{y}_{\mathcal{M}}\right)$ where $\mathbf{y}_{E_{0: k}}$ represent the set of sensor readings gathered by the sensors mounted on the robots from environmental features from time step 0 up to time step $k$, and $\mathbf{y}_{\mathcal{M}}$ represents the stochastic map of the previously mapped navigation area. In the sequel, the EKF algorithm [2] is used as the core estimation technique.

\section{A. EKF Prediction Step}

The EKF-prediction step propagates the state of the robot formation from time step $k-1$ to time step $k$ by using deadreckoning measurements:

$$
\mathbf{x}_{\mathcal{R}_{k}} \simeq \mathbf{F}_{k-1} \mathbf{x}_{\mathcal{R}_{k-1}}+\mathbf{v}_{k-1}
$$

where the block-diagonal matrix $\mathbf{F}_{k-1}$ represents the jacobian matrix of the linearized motion equations of the robot team and $\mathbf{v}_{k-1}$ represents a zero-mean white noise sequence with a block-diagonal covariance matrix $\mathbf{Q}_{k-1}$. Eq. (2) provides estimates for the state vector $\hat{\mathbf{x}}_{\mathcal{R}_{k \mid k-1}}$ and its associated covariance matrix $\mathbf{P}_{\mathcal{R}_{k \mid k-1}}$.

\section{B. EKF Update Step}

At time step $k$, robust data association provides the algorithm with a set of jointly consistent pairs $\left(\mathcal{E}_{k}, \mathcal{F}_{k}\right)$ formed by sensor observations and map features respectively. Due to the inherent nonlinearities, a linearized measurement equation is used within the EKF-update step:

$$
\mathbf{z}_{k} \simeq \mathbf{H}_{k} \mathbf{x}_{\mathcal{R}_{k}}+\mathbf{G}_{k} \mathbf{y}_{k}
$$

where $\mathbf{H}_{k}$ and $\mathbf{G}_{k}=\left(\mathbf{G}_{\mathcal{E}_{k}} \quad \mathbf{G}_{\mathcal{F}_{k}}\right)$ are the Jacobian matrices of the linearized measurement equations with respect to the state vector $\mathbf{x}_{\mathcal{R}_{k}}$ and with respect to the matched sensor observations $\mathbf{y}_{k}$ respectively, with:

$$
\mathbf{y}_{k}=\left(\begin{array}{c}
\mathbf{y}_{\mathcal{E}_{k}} \\
\mathbf{y}_{\mathcal{F}_{k}}
\end{array}\right) \sim \mathcal{N}\left(\left(\begin{array}{c}
\hat{\mathbf{y}}_{\mathcal{E}_{k}} \\
\hat{\mathbf{y}}_{\mathcal{F}_{k}}
\end{array}\right),\left(\begin{array}{cc}
\mathbf{P}_{\mathcal{E}_{k}} & \mathbf{0} \\
\mathbf{0} & \mathbf{P}_{\mathcal{F}_{k}}
\end{array}\right)\right)
$$

and $\mathbf{y}_{\mathcal{F}_{k}} \subset \mathbf{y}_{\mathcal{M}}$ a subset of map features from the a priori stochastic map.

The classical EKF update equations provide estimates for the state vector $\hat{\mathbf{x}}_{\mathcal{R}_{k \mid k}}$ and its associated covariance matrix $\mathbf{P}_{\mathcal{R}_{k \mid k}}$ using the filter gain given by:

$$
\mathbf{K}_{k}=\mathbf{P}_{\mathcal{R}_{k \mid k-1}} \mathbf{H}_{k}^{T}\left(\mathbf{H}_{k} \mathbf{P}_{\mathcal{R}_{k \mid k-1}} \mathbf{H}_{k}^{T}+\mathbf{R}_{k}\right)^{-1}
$$

with,

$$
\mathbf{R}_{k}=\mathbf{G}_{\mathcal{E}_{k}} \mathbf{P}_{\mathcal{E}_{k}} \mathbf{G}_{\mathcal{E}_{k}}^{T}+\mathbf{G}_{\mathcal{F}_{k}} \mathbf{P}_{\mathcal{F}_{k}} \mathbf{G}_{\mathcal{F}_{k}}^{T}
$$

The estimated state vector and its associated covariance matrix are given by,

$$
\begin{aligned}
\hat{\mathbf{x}}_{\mathcal{R}_{k \mid k}} & =\hat{\mathbf{x}}_{\mathcal{R}_{k \mid k-1}}+\mathbf{K}_{k}\left(\mathbf{z}_{k}-\hat{\mathbf{z}}_{k}\right) \\
\mathbf{P}_{\mathcal{R}_{k \mid k}} & =\left(\mathbf{I}-\mathbf{K}_{k} \mathbf{H}_{k}\right) \mathbf{P}_{\mathcal{R}_{k \mid k-1}}
\end{aligned}
$$

\section{Simulation Results: Inconsistency}

A simulation experiment was conducted to analyze the consistency of a direct implementation of the EKF algorithm based on the aforementioned problem formulation. A 3-robot formation was commanded to navigate through a 200-m loop-trajectory within a previously mapped (2D point-based stochastic map) navigation area. Each vehicle was equipped with a range-bearing sensor capable of observing the available environmental features. As a measure of consistency [2] a statistical test based on the Normalized Estimation Error Squared (NEES) was used,

$$
\mathrm{NEES}=\left(\mathbf{x}_{\mathcal{R}_{k}}-\hat{\mathbf{x}}_{\mathcal{R}_{k}}\right)^{T} \mathbf{P}_{\mathcal{R}}^{-1}\left(\mathbf{x}_{\mathcal{R}_{k}}-\hat{\mathbf{x}}_{\mathcal{R}_{k}}\right) \leq \chi_{r, 1-\alpha}^{2}
$$

where $\chi_{r, 1-\alpha}^{2}$ is a threshold obtained from the $\chi^{2}$ distribution with $r=\operatorname{dim}\left(\mathbf{x}_{\mathcal{R}_{k}}\right)$ degrees of freedom, and $\alpha$ the desired significance level (usually 0.05).

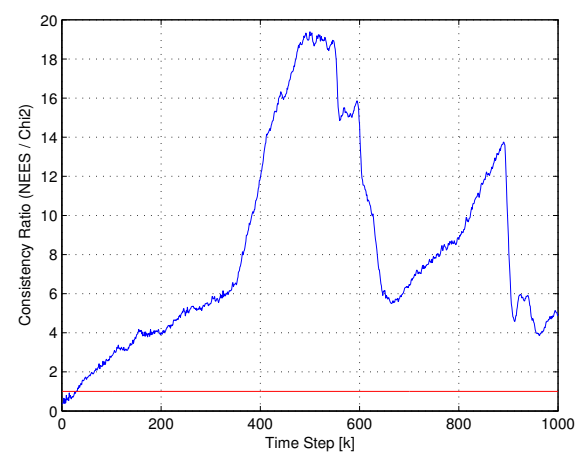

Fig. 1. Inconsistency of the estimated robot formation localization with a direct implementation of the EKF algorithm. The consistency ratio NEES $/ \chi_{r, 1-\alpha}^{2}$ should be less than one. The average of 10 Monte Carlo runs is depicted.

Figure 1 plots the consistency ratio $\left(\mathrm{NEES} / \chi_{r, 1-\alpha}^{2}\right)$ for the sequence of time steps of the experiment illustrating a problem of inconsistency in the estimated solution of this direct implementation of the EKF algorithm. In the following section we take a closer view of the results and a modified EKF formulation is proposed to drive the robot formation estimated localization towards consistency. 


\section{Measurement-Differencing EKF-BASEd LOCALIZATION OF THE ROBOT FORMATION}

A close view of the robot formation localization problem within an a priori stochastic map, supported by the simulation results obtained in the previous section, suggest a reformulation of the EKF algorithm taking into account the statistical dependencies between the observations $\mathbf{y}_{\mathcal{F}_{k-1}}$ and $\mathbf{y}_{\mathcal{F}_{k}}$ (both subsets of $\mathbf{y}_{\mathcal{M}}$ ) used within the EKF update step in two consecutive time steps $k-1$ and $k$.

Given the set of matched map features of two consecutive time instants $\mathbf{y}_{\mathcal{F}_{k-1}}$ and $\mathbf{y}_{\mathcal{F}_{k}}$, their statistical dependencies are expressed by a linear transformation,

$$
\mathbf{y}_{\mathcal{F}_{k}}=\mathbf{F}_{C_{k}} \mathbf{y}_{\mathcal{F}_{k-1}}+\mathbf{w}_{\omega_{k}}
$$

where $\mathbf{F}_{C_{k}}$ is row-selection matrix and $\mathbf{w}_{\omega_{k}}$ is a white noise measurement sequence with covariance matrix $\mathbf{P}_{\mathbf{w}_{\omega_{k}}}$. Equation (9) defines a colored measurement noise sequence, which together with eqs. (2) and (3) completely reformulates the problem at hand.

The first approach reported in the literature which considers the existence of a colored measurement noise sequence within the EKF framework dates back to the works of Bryson et al. [4] where the state vector was augmented with the colored error terms. Later work pointed out relevant numerical problems of this approach mainly due to nulluncertainty observations and ill-conditioned transition matrices. Current practical approaches [2], [11] concern the socalled measurement differencing technique, which provides an efficient and mathematically sound method to remove the time-correlated portion of the measurement errors. We extend previous work by others in the field of filtering theory by formulating the robot formation localization problem in SLAM as a measurement differencing based EKF algorithm to whiten the originally colored measurement noise sequence defined in eq. (9).

\section{A. Whitening the Measurement Equation}

Let $\mathbf{r}_{k}$ represent the measurement considered within the EKF-update step at time $k$, derived from the real measurements $\mathbf{z}_{k-1}$ and $\mathbf{z}_{k}$ obtained at two consecutive time instants as,

$$
\mathbf{r}_{k} \triangleq \mathbf{z}_{k}-\boldsymbol{\Lambda}_{k} \mathbf{z}_{k-1}
$$

where matrix $\boldsymbol{\Lambda}_{k}$ is chosen such that $\left\{\mathbf{r}_{k}, 0 \leq k<\infty\right\}$ approaches a discrete-time white-noise driven stochastic process [2], [11].

Following the derivation of the appendix measurement $\mathbf{r}_{k}$ can be rewritten as,

$$
\mathbf{r}_{k} \simeq \mathbf{H}_{k}^{*} \mathbf{x}_{\mathcal{R}_{k}}+\mathbf{w}_{k}
$$

where,

$$
\mathbf{H}_{k}^{*}=\mathbf{H}_{k}-\boldsymbol{\Lambda}_{k} \mathbf{H}_{k-1} \mathbf{F}_{k-1}^{-1}
$$

and the white noise sequence $\mathbf{w}_{k}$, with covariance matrix $\mathbf{P}_{\mathbf{w}_{k}}$, is given by,

$$
\begin{aligned}
\mathbf{w}_{k} & =\mathbf{G}_{\mathcal{E}_{k}} \mathbf{y}_{\mathcal{E}_{k}}-\boldsymbol{\Lambda}_{k} \mathbf{G}_{\mathcal{E}_{k-1}} \mathbf{y}_{\mathcal{E}_{k-1}}+\mathbf{G}_{\mathcal{F}_{k}} \mathbf{w}_{\omega_{k}} \\
& +\boldsymbol{\Lambda}_{k} \mathbf{H}_{k-1} \mathbf{F}_{k-1}^{-1} \mathbf{v}_{k-1}
\end{aligned}
$$

Matrix $\boldsymbol{\Lambda}_{k}$ is computed (see appendix) such that the timecorrelated components of the measurement $\mathbf{r}_{k}$ are removed,

$$
\boldsymbol{\Lambda}_{k} \simeq \mathbf{G}_{\mathcal{F}_{k}} \mathbf{F}_{C_{k}} \mathbf{G}_{\mathcal{F}_{k-1}}^{T}\left(\mathbf{G}_{\mathcal{F}_{k-1}} \mathbf{G}_{\mathcal{F}_{k-1}}^{T}\right)^{-1}
$$

Note that previous works [2], [11], under the linearity assumption both in the motion and measurement equations, reported that $\boldsymbol{\Lambda}_{k}=\mathbf{F}_{C_{k}}$, being a particular case of the more general result provided in this paper. In our case, the existence of matrix $\boldsymbol{\Lambda}_{k}$ has been verified for the cases of 2D-point and 2D-segment based stochastic maps.

Finally, eq. (13) introduce a cross-correlation term between $\mathbf{w}_{k}$ and $\mathbf{v}_{k-1}$, namely,

$$
\mathbf{C}_{k}=E\left[\mathbf{v}_{k-1} \mathbf{w}_{k}^{T}\right]=\mathbf{Q}_{k-1}\left(\boldsymbol{\Lambda}_{k} \mathbf{H}_{k-1} \mathbf{F}_{k-1}^{-1}\right)^{T}
$$

which is introduced in the EKF algorithm following [3] using the filter gain,

$$
\begin{aligned}
\mathbf{K}_{k}= & \left(\mathbf{P}_{\mathcal{R}_{k \mid k-1}} \mathbf{H}_{k}^{* T}+\mathbf{C}_{k}\right) . \\
& \left(\mathbf{H}_{k}^{*} \mathbf{P}_{\mathcal{R}_{k \mid k-1}} \mathbf{H}_{k}^{* T}+\mathbf{P}_{\mathbf{w}_{k}}+\mathbf{H}_{k}^{*} \mathbf{C}_{k}+\mathbf{C}_{k}^{T} \mathbf{H}_{k}^{* T}\right)^{-1}
\end{aligned}
$$

and the update of the estimated covariance matrix given by,

$$
\begin{gathered}
\mathbf{P}_{\mathcal{R}_{k \mid k}}=\mathbf{P}_{\mathcal{R}_{k \mid k-1}}-\mathbf{K}_{k}\left(\mathbf{H}_{k}^{*} \mathbf{P}_{\mathcal{R}_{k \mid k-1}} \mathbf{H}_{k}^{* T}+\mathbf{P}_{\mathbf{w}_{k}}\right. \\
\left.+\mathbf{H}_{k}^{*} \mathbf{C}_{k}+\mathbf{C}_{k}^{T} \mathbf{H}_{k}^{* T}\right) \mathbf{K}_{k}^{T}
\end{gathered}
$$

The computational cost of the modified EKF algorithm does not appreciably increase over the direct implementation because the dimensions of the matrices involved are the same.

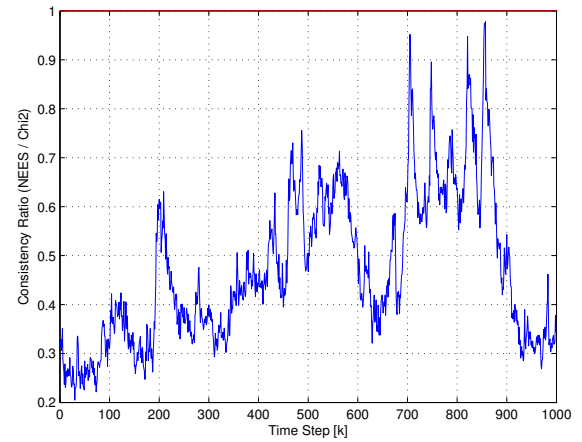

Fig. 2. Improvement in the consistency of the estimated robot formation localization with a measurement-differencing based EKF algorithm. The average of 10 Monte Carlo runs is depicted.

Figure 2 plots the consistency ratio $\left(\mathrm{NEES} / \chi_{r, 1-\alpha}^{2}\right.$ ) for the sequence of time steps of the experiment for the implementation of the measurement differencing algorithm proposed in this section. In this case, the modified EKF algorithm provides a consistent solution for the robot formation estimated localization. 


\section{EXPERIMENTAL RESUlts}

The measurement differencing EKF-based localization algorithm has been tested both in the multi-robot simulation platform Player/Stage [12] and in real experiments with a 3-robot Pioneer 3-AT team in a triangular-shaped formation scheme.

\section{A. Experiments in Player/Stage}

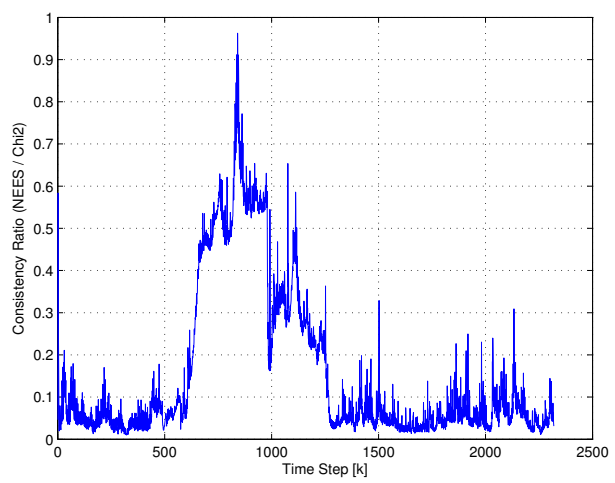

Fig. 3. Consistency of the estimated robot formation localization with a measurement-differencing based EKF algorithm in the Player/Stage experiments. The average of 10 Monte Carlo runs is depicted.

A first set of experiments have been conducted within the rapid prototyping tool Player/Stage [12] which allows code development and testing in similar conditions as to those subsequently faced in the real scenario but constraint to the information provided by simulated motion control and data acquisition.

The formation was commanded a 100-m loop trajectory within a previously available segment-based stochastic map. Thanks to the availability of ground-truth, the consistency of the proposed algorithm in this quasi-real scenario was verified as the consistency ratio plot of fig. (3) highlights. Also, in figs. 4 the frontal, lateral and angular errors for each robot in the formation are displayed together with their associated 2- $\sigma$ uncertainty bounds. In all the cases the estimated errors are within the computed bounds.

\section{B. Experiments with the Pioneer 3-AT Robots}

Real experiments have been conducted by using a 3-robot triangular-shaped formation of Pioneer 3-AT vehicles. Fig. (5) depicts the initial localization of the vehicles within an indoor environment. The formation was commanded to reach a distant goal location (about 40-m from the starting location) while avoiding obstacles and adapting its shape to the environment. The robot leader plans a safe path to the goal destination and the robot slaves follow the leader while maintaining the desired formation topology. Communication among the vehicles of the formation is provided by a realtime wireless multihop protocol implemented in a centralized mode where robot slaves sent both the sensors observations and the commanded velocities to the robot leader. The robot
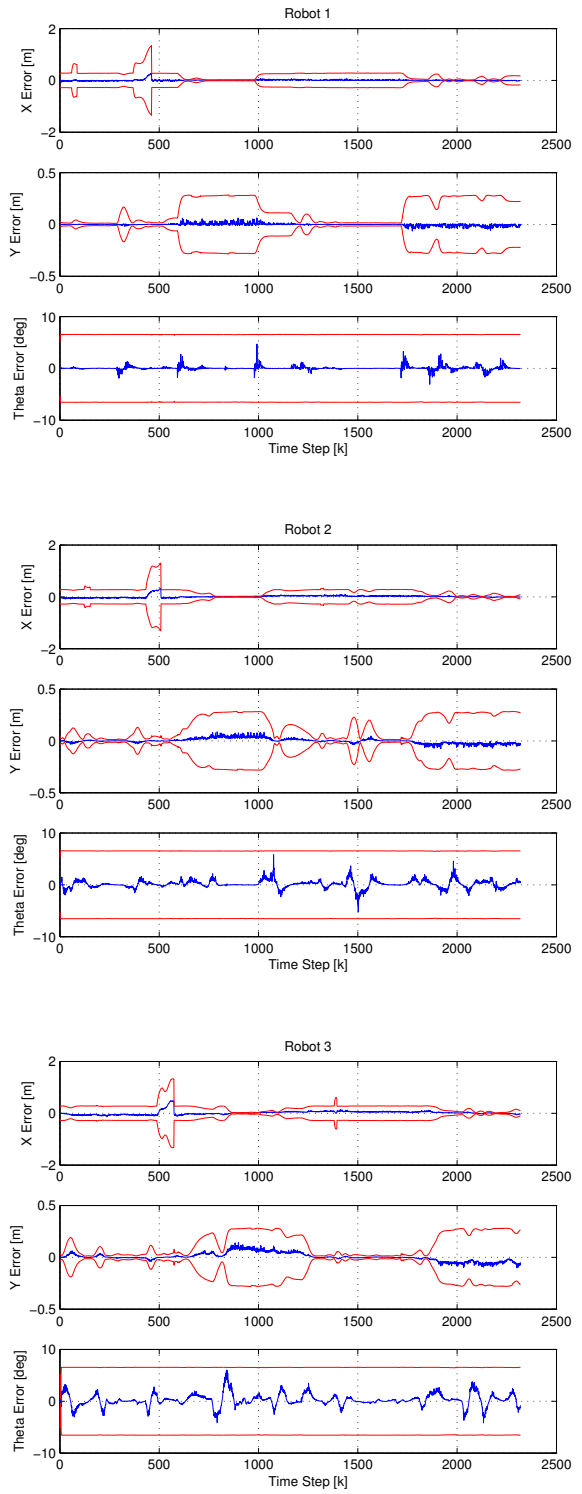

Fig. 4. Experimental results obtained in the Player/Stage setting. Frontal, lateral and angular errors for each vehicle in the 3-robot triangular-shaped formation and their associated 2- $\sigma$ uncertainty bounds are shown: Robot leader (top) and robot slaves (middle and bottom)

leader executes the localization algorithm and it communicates the estimated poses to each robot slave ([17] and reference therein).

Initially, a segment-based stochastic map of the navigation area (fig. 5, middle) is obtained by using the information provided by the 2-D laser scanned mounted in one of the vehicle (in our case, and without lose of generality, the robot leader) which previously had explored the environment. Then, the global localization of the vehicles (fig. 5, bottom) is computed by the algorithms reported in [7].

Figure (6) shows the estimated localization of each of the vehicles of the formation at four different time steps along the planned trajectory towards the goal destination. Even though ground-truth was not available during the execution 


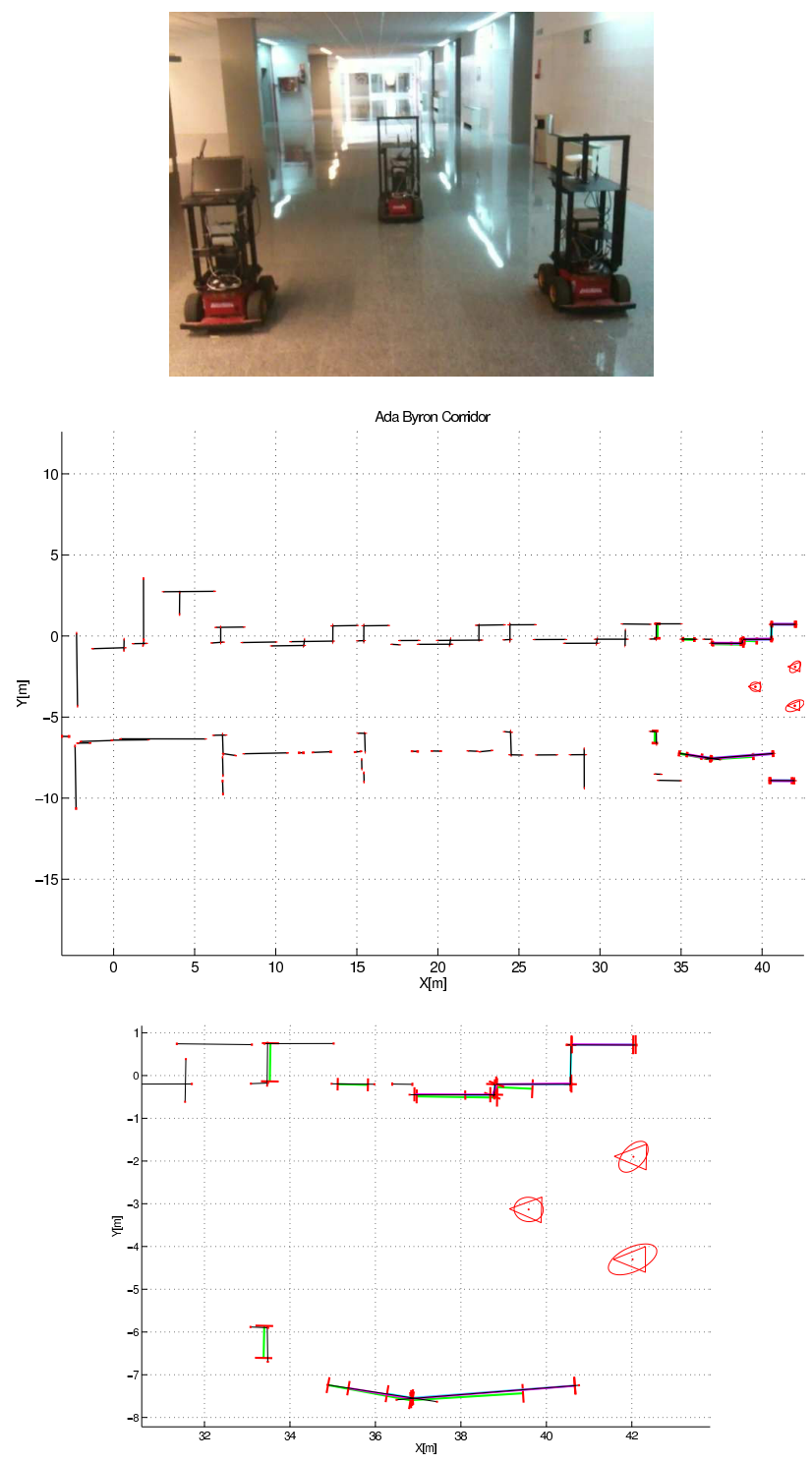

Fig. 5. Initial setting of the robot formation in a real experiment: 3robot triangular-shaped formation (top), segment-based stochastic map of the navigation area (middle), and EIF-based initial localization of the robot formation within the map(bottom, with location uncertainty magnified $x 5$ ).

of the real experiments, the figure highlights the compatibility between the previously available stochastic map and the segmented sensor readings plotted wrt the estimated vehicles localization.

\section{CONCLUSiOnS}

The work described in this paper concerned the utilization of feature-based stochastic maps for robot formation localization. From the Bayesian perspective, the classical EKF algorithm was initially formulated by considering the motion models of each vehicle within the formation and the environmental observations gathered by the exteroceptive sensors mounted on the vehicles. The inconsistency of the direct implementation of the EKF prediction and update equations for the problem at hand is reported. A closer view to the algorithm hypotheses is conducted suggesting the timecorrelated nature of the sequence of measurements considered in the previous, direct implementation. A solution, based on the measurement differencing technique, already reported in the filtering literature is adapted to the robot formation localization problem in SLAM and its implementation shows how the modified EKF estimation is driven towards consistency. Simulated and real experiments with a 3-robot triangular-shaped formation are reported to demonstrate the applicability of the adoptive filtering approach.

Future work moves towards decentralized schemes, both in terms of localization and control of the members of the formation. An intense cooperation among localization, planning, obstacle avoidance and SLAM is also being investigated. Finally, more general nonlinear, non-Gaussian Bayesian approaches are being analyzed within the general framework of robot formations.

\section{ACKNOWLEDGEMENTS}

The authors sincerely thanks to Pablo Urcola and Domenico Sicignano, from the University of Zaragoza, for their help during the experimentation with the Pioneer robots.

\section{APPENDIX}

Let $\mathbf{r}_{k}$ be expressed as,

$$
\mathbf{r}_{k} \triangleq \mathbf{z}_{k}-\Lambda_{k} \mathbf{z}_{k-1}
$$

Substituting the observations $\mathbf{z}_{k}$ and $\mathbf{z}_{k-1}$ by their linearized expressions given by eq. (3),

$$
\begin{aligned}
\mathbf{r}_{k} \simeq & \mathbf{H}_{k} \mathbf{x}_{\mathcal{R}_{k}}+\mathbf{G}_{k} \mathbf{y}_{k} \\
& -\boldsymbol{\Lambda}_{k}\left(\mathbf{H}_{k-1} \mathbf{x}_{\mathcal{R}_{k-1}}+\mathbf{G}_{k-1} \mathbf{y}_{k-1}\right)
\end{aligned}
$$

Considering the form of the observations given by eq. (4) we have,

$$
\begin{aligned}
\mathbf{r}_{k} \simeq & \mathbf{H}_{k} \mathbf{x}_{\mathcal{R}_{k}}+\mathbf{G}_{\mathcal{E}_{k}} \mathbf{y}_{\mathcal{E}_{k}}+\mathbf{G}_{\mathcal{F}_{k}} \mathbf{y}_{\mathcal{F}_{k}} \\
& -\boldsymbol{\Lambda}_{k}\left(\mathbf{H}_{k-1} \mathbf{x}_{\mathcal{R}_{k-1}}+\mathbf{G}_{\mathcal{E}_{k-1}} \mathbf{y}_{\mathcal{E}_{k-1}}+\mathbf{G}_{\mathcal{F}_{k-1}} \mathbf{y}_{\mathcal{F}_{k-1}}\right)
\end{aligned}
$$

Rearranging the system equation given by eq. (2) following the approach described in [11] to avoid time-latency leads to,

$$
\mathbf{x}_{\mathcal{R}_{k-1}} \simeq \mathbf{F}_{k-1}^{-1} \mathbf{x}_{\mathcal{R}_{k}}-\mathbf{F}_{k-1}^{-1} \mathbf{v}_{k-1}
$$

therefore,

$$
\begin{aligned}
\mathbf{r}_{k} \simeq & \left(\mathbf{H}_{k}-\boldsymbol{\Lambda}_{k} \mathbf{H}_{k-1} \mathbf{F}_{k-1}^{-1}\right) \mathbf{x}_{\mathcal{R}_{k}}+\boldsymbol{\Lambda}_{k} \mathbf{H}_{k-1} \mathbf{F}_{k-1}^{-1} \mathbf{v}_{k-1} \\
& +\mathbf{G}_{\mathcal{E}_{k}} \mathbf{y}_{\mathcal{E}_{k}}-\boldsymbol{\Lambda}_{k} \mathbf{G}_{\mathcal{E}_{k-1}} \mathbf{y}_{\mathcal{E}_{k-1}} \\
& +\mathbf{G}_{\mathcal{F}_{k}} \mathbf{y}_{\mathcal{F}_{k}}-\boldsymbol{\Lambda}_{k} \mathbf{G}_{\mathcal{F}_{k-1}} \mathbf{y}_{\mathcal{F}_{k-1}}
\end{aligned}
$$

Substituting the linear relation between $\mathbf{y}_{\mathcal{F}_{k}}$ and $\mathbf{y}_{\mathcal{F}_{k-1}}$ given by eq. (9) results in,

$$
\begin{aligned}
\mathbf{r}_{k} \simeq & \left(\mathbf{H}_{k}-\boldsymbol{\Lambda}_{k} \mathbf{H}_{k-1} \mathbf{F}_{k-1}^{-1}\right) \mathbf{x}_{\mathcal{R}_{k}}+\boldsymbol{\Lambda}_{k} \mathbf{H}_{k-1} \mathbf{F}_{k-1}^{-1} \mathbf{v}_{k-1} \\
& +\mathbf{G}_{\mathcal{E}_{k}} \mathbf{y}_{\mathcal{E}_{k}}-\boldsymbol{\Lambda}_{k} \mathbf{G}_{\mathcal{E}_{k-1}} \mathbf{y}_{\mathcal{E}_{k-1}}+\mathbf{G}_{\mathcal{F}_{k}} \mathbf{w}_{\omega_{k}} \\
& +\left(\mathbf{G}_{\mathcal{F}_{k}} \mathbf{F}_{C_{k}}-\boldsymbol{\Lambda}_{k} \mathbf{G}_{\mathcal{F}_{k-1}}\right) \mathbf{y}_{\mathcal{F}_{k-1}}
\end{aligned}
$$

which can finally be expressed as,

$$
\mathbf{r}_{k} \simeq \mathbf{H}_{k}^{*} \mathbf{x}_{\mathcal{R}_{k}}+\mathbf{w}_{k}
$$

with,

$$
\mathbf{H}_{k}^{*}=\mathbf{H}_{k}-\boldsymbol{\Lambda}_{k} \mathbf{H}_{k-1} \mathbf{F}_{k-1}^{-1}
$$


and,

$$
\begin{aligned}
\mathbf{w}_{k} & =\mathbf{G}_{\mathcal{E}_{k}} \mathbf{y}_{\mathcal{E}_{k}}-\boldsymbol{\Lambda}_{k} \mathbf{G}_{\mathcal{E}_{k-1}} \mathbf{y}_{\mathcal{E}_{k-1}}+\mathbf{G}_{\mathcal{F}_{k}} \mathbf{w}_{\omega_{k}} \\
& +\boldsymbol{\Lambda}_{k} \mathbf{H}_{k-1} \mathbf{F}_{k-1}^{-1} \mathbf{v}_{k-1}
\end{aligned}
$$

and, matrix $\boldsymbol{\Lambda}_{k}$ is computed such that the time-correlated components from the evolution of the measurement $\mathbf{r}_{k}$ are removed, thus is,

$$
\mathbf{G}_{\mathcal{F}_{k}} \mathbf{F}_{C_{k}}-\boldsymbol{\Lambda}_{k} \mathbf{G}_{\mathcal{F}_{k-1}} \simeq \mathbf{0}
$$

thus,

$$
\begin{gathered}
\boldsymbol{\Lambda}_{k} \simeq \mathbf{G}_{\mathcal{F}_{k}} \mathbf{F}_{C_{k}} \mathbf{G}_{\mathcal{F}_{k-1}}^{T}\left(\mathbf{G}_{\mathcal{F}_{k-1}} \mathbf{G}_{\mathcal{F}_{k-1}}^{T}\right)^{-1} \\
\text { REFERENCES }
\end{gathered}
$$

[1] A. Bahr, M. R. Walter and J. J. Leonard, "Consistent Cooperative Localization" Proc. IEEE Int. Conf. on Robotics and Automation, Kobe, Japan, May 12-17, 2009.

[2] Y. Bar-Shalom, X. Rong Li, T. Kirubarajan, "Estimation with Applications to Tracking and Navigation", Wiley and Sons, May 2001.

[3] R. G. Brown and P. Y. C. Hwang, "Introduction to Random Signals and Applied Kalman Filtering", Wiley and sons, 2nd Edition, New York, 1992.

[4] A. E. Bryson and D. E. Johansen, "Linear Filtering for TimeVarying Systems Using Measurements Containing Colored Noise", IEEE Trans. on Automatic Control, January 1968.

[5] G. P. Huang, N. Trawny, A. I. Mourikis and S. I. Roumeliotis, "On the Consistency of Multi-robot Cooperative Localization", Proc. of Robotics Science and Systems, 2009, Seattle, Washington.

[6] R. Kurazume, S. Nagata and S. Hirose, "Cooperative positioning with multiple robots", in Proc. of the IEEE Int. Conf. in Robotics and Automation, Los Alamitos, CA, 1994, pp. 1250-1257.

[7] L. M. Paz, P. Piniés, J. Neira and J.D. Tardós, "Global Localization in SLAM in Bilinear Time", in Proc. of the IEEE/RSJ Int. Conf. on Intelligent Robots and Systems, 2005, Edmonton, Canada, pp. 655661.

[8] A. I. Mourikis, S. I. Roumeliotis, and J. W. Burdick, "SC-KF Mobile Robot Localization: A Stochastic Cloning-Kalman Filter for Processing Relative-State Measurements", Robotics Science and Systems, 2009, Seattle, Washington.

[9] J. Neira, J. D. Tardós and J. A. Castellanos, "Linear Time Vehicle Relocation in SLAM", Proc. IEEE Int. Conf. on Robotics and Automation, 2003, pp. 427-433, Taipei, Taiwan.

[10] I. M. Rekleitis, G. Dudek, and E. Milios, "Multi-robot exploration of an unknown environment, efficiently reducing the odometry error" Proc. of the Fifteenth International Joint Conference on Artificial Intelligence, Nagoya, Japan, Aug. 23-29 1997, pp. 1340-1345.

[11] M. G. Petovello, K. O'Keefe, G. Lachapelle and M. E. Cannon, "Consideration of Time-correlated Errors in a Kalman filter applicable to GNSS", in Journal of Geodesy, 83:51-56, 2009.

[12] http://playerstage.sourceforge.net/index.php

[13] S. I. Roumeliotis and G. A. Bekey, "Collective Localization: A distributed Kalman filter approach to localization of groups of mobile robots", Proc. of the IEEE Int. Conf. on Robotics and Automation, San Francisco, CA, April 2000.

[14] A. C. Sanderson, "A distributed algorithm for cooperative navigation among multiple mobile robots", Advanced Robotics, Vol. 12, No. 4, 1998, pp. 335-349.

[15] A. C. Schultz and W. Adams, "Continuous Localization Using Evidence Grids", Proc. of the IEEE Int. Conf. on Robotics and Automation, Leuven, Belgium, May 1998.

[16] S. Thrun, "Learning metric-topological maps for indoor mobile robot navigation", Artificial Intelligence, Vol. 99, No. 1, 1998, pp. 21-71.

[17] P. Urcola, L. Montano, "Cooperative robot team navigation strategies based on an environmental model", Proc. of the IEEE/RSJ International Conference on Intelligent Robots and Systems, St. Louis, MO, Oct 11-15, 2009.

[18] URUS: "Ubiquitous Networking Robotics in Urban Settings", European project IST-1-045062-URUS-STP. More information available at the project website: http://urus.upc.es
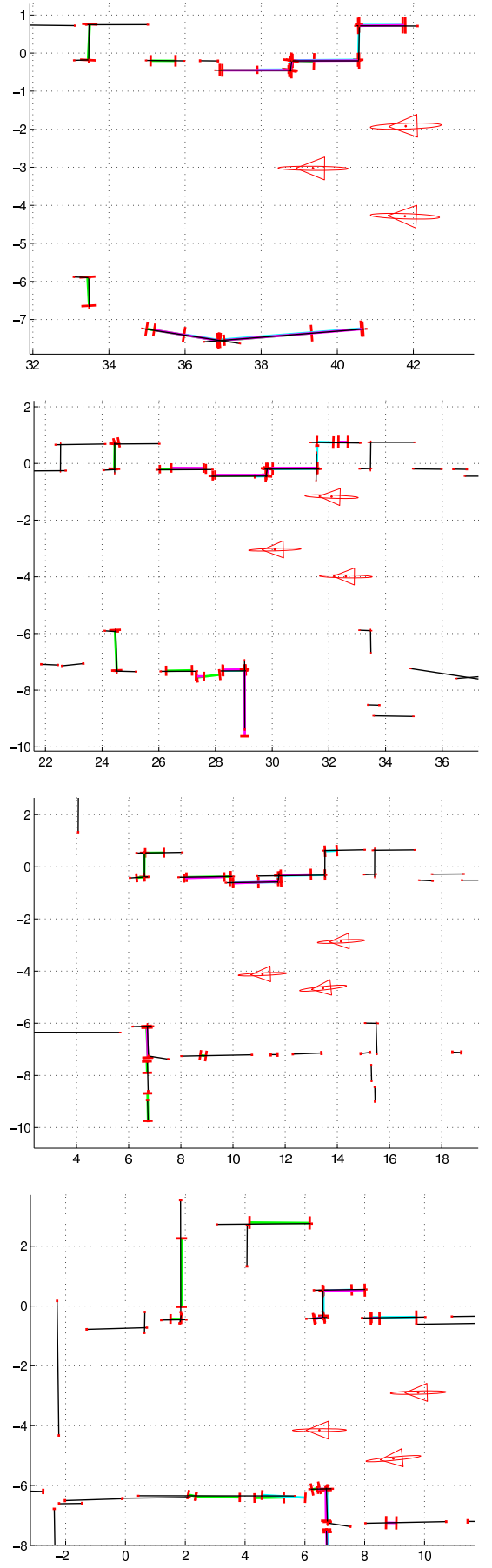

Fig. 6. Four snapshots (from top to bottom) of the estimated localization of the members of the robot formation within the stochastic map (plotted in black). The segmented sensor observations are plotted in green, blue and magenta and red lines represent the segments uncertainties. Robot uncertainties ellipses are magnified by $\mathrm{x} 5$. 